\title{
Design Optimization of Coulomb Blockade Devices
}

\author{
H.-O. MÜLLER*, D. A. WILLIAMS ${ }^{\dagger}$ and H. MIZUTA ${ }^{\ddagger}$ \\ Hitachi Cambridge Laboratory, Cavendish Laboratory, Madingley Road, Cambridge, CB3 OHE, UK
}

\begin{abstract}
We investigate the design of a Coulomb blockade device consisting of a rectangular array of quantum dots or ultrasmall metallic islands with regard to its stability against geometric size disorder and offset charges. To simulate the device operation we perform a statistical analysis of the Coulomb blockade voltage which results in practical design rules.
\end{abstract}

Keywords: Coulomb blockade; Device physics; Quantum dot array; Size disorder; Background charge

PACS: 73.23.Hk, 85.30.Wx, 07.05.Tp

\section{INTRODUCTION}

For digital applications the complete current suppression offered by Coulomb blockade (CB) [1] is very attractive as it would lead to lower power consumption. This complete suppression is strictly speaking only theoretical, since it results from a first-order perturbation expansion at zero temperature. Therefore, most CB devices have to tackle problems of parasitic currents caused by thermally increased conduction, cotunneling, or time-varying fields. In addition there are technological difficulties, namely geometric disorder of the device, and offset charges.

In order to tackle these problems, instead of a single quantum dot or ultrasmall metallic island, several proposed and realized CB devices are based on arrays, such as the thermometer [2], the current standard [3], and the silicon CB memory [4]. Also two-dimensional arrays haven been investigated theoretically [5], by means of simulation $[6,7]$, and experimentally $[8,9]$. The overall blockade voltage, $V_{b}$, is used as a figure of merit for CB devices, and is a measure of the suppressability of current flow through a dot or an array.

Here we use computer simulation of the blockade voltage $V_{b}$ to understand the effect of array size, geometric disorder, and offset charges on its mean value $\left\langle V_{b}\right\rangle$ and its variation $\left\langle\left\langle V_{b}\right\rangle\right\rangle .\left\langle V_{b}\right\rangle$ is used to study the feasibility of a specific device, to plan the outer circuitry, and to develop a suitable bias regime for this circuitry. $\left\langle\left\langle V_{b}\right\rangle\right\rangle$ is connected

\footnotetext{
*e-mail: hom@ise.ch

†e-mail: williams@phy.cam.ac.uk

${ }^{\ddagger}$ Corresponding author. e-mail: mizuta@phy.cam.ac.uk
} 
with questions of reliability, device performance, and operating margins. We investigate arrays of size $1 \times 1$ (single $\operatorname{dot} /$ island) to $5 \times 5$, and ten samples per degree of disorder or offset-charge level (defined below). The method of our simulation is described in great detail elsewhere [10].

Real arrays can display geometric size disorder and offset charge disorder. Size disorder is simulated by varying the two lateral dimensions of vertically thin quantum dots independently of each other. The center of the dot is kept fixed, defining an upper limit to this variation, in order to avoid dot overlap. The degree of size disorder is measured as a percentage of this maximal variation. In our example the nominal dot size is $5 \mathrm{~nm}$ and the maximum variation is $2 \mathrm{~nm}$. For dot-todot distances larger than $2 \mathrm{~nm}$, tunneling in the $\mathrm{CB}$ regime is strongly suppressed.

Background charges can influence additional charge on weakly coupled quantum dots and so offset charge disorder is investigated by simulating randomly induced charge on the dot in the array. The values of this charge are restricted to an interval of $1 e$, by convention [ $-e / 2: e / 2]$, because the response of $\mathrm{CB}$ devices is periodic in $e$. We measure the influence of offset charges by the charge they might influence on the dot. This range is again presented as a percentage of the maximal range $[-e / 2: e / 2]$.

For clarity we discuss only a fraction of the obtained data. We leave out the case of

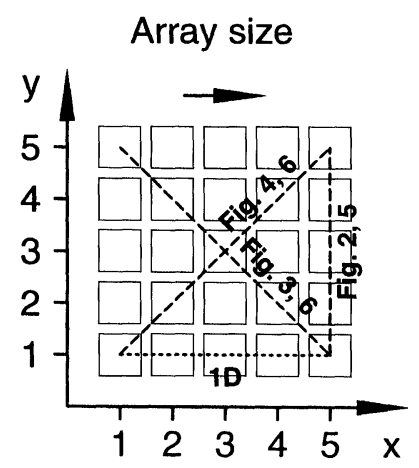

FIGURE 1 Array sizes studied in this paper. We investigate the following transitions: $1 \times 1$ to $1 \times 5,1 \times 5$ to $5 \times 1$, and $1 \times 1$ to $5 \times 5$ as indicated by the different dashed lines. The current flows in the $x$ direction. one-dimensional arrays, since this is extensively described in the contemporary literature [11]. As depicted in Figure 1 we concentrate on the width dependence of arrays and look at the transition of short and wide arrays to long and narrow ones. Since square-like arrays are of special interest, we study them as well.

\section{RESULTS}

\subsection{Size Disorder as Function of the Array Width}

Figure 2 displays our results for $\left\langle V_{b}\right\rangle$ and $\left\langle\left\langle V_{b}\right\rangle\right\rangle$ for different array widths and for different levels of size disorder. There is a slight increase of $\left\langle V_{b}\right\rangle$ with increasing array width. The data for different degrees of disorder save the highest are hardly different. For $90 \%$ disorder we observe a $15 \%$ increase in $\left\langle V_{b}\right\rangle$.

The $\left\langle\left\langle V_{b}\right\rangle\right\rangle$ dependence (Fig. 2(b)) does not show any conclusive behavior as function of the array width. However, there is a drastic increase with the level of disorder (logarithmic scale). The ratio $\left\langle\left\langle V_{b}\right\rangle\right\rangle:\left\langle\left\langle V_{b}\right\rangle\right\rangle$ provides an estimate of the accuracy of the study of $\left\langle V_{b}\right\rangle$ and our analysis yields $\left\langle V_{b}\right\rangle \gg$ $\left\langle\left\langle V_{b}\right\rangle\right\rangle$. We obtain similar levels of for $\left\langle\left\langle V_{b}\right\rangle\right\rangle$ other geometries (see for example Fig. 3(b)).

How can we understand these results? $\left\langle V_{b}\right\rangle$ depends on the array width and size disorder. Generally, disordered arrays display charge pinning $[6,7]$ which yields a $\left\langle V_{b}\right\rangle$ increase. In wider arrays charge pinning is increased due to the electrostatic coupling of pinned charges and
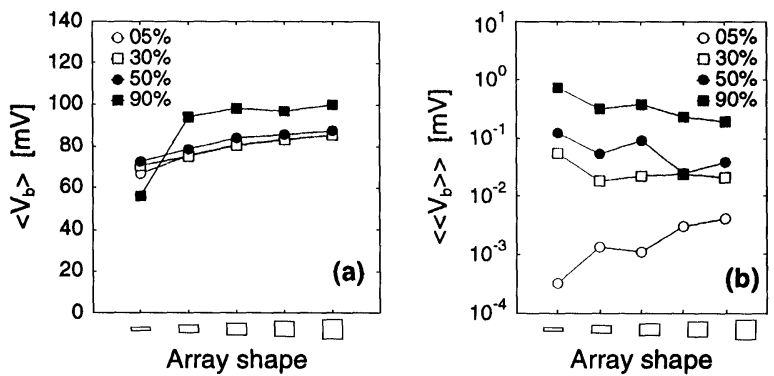

FIGURE 2 Array width dependence of $\left\langle V_{b}\right\rangle$ and $\left\langle\left\langle V_{b}\right\rangle\right\rangle$ for different values of size disorder. 

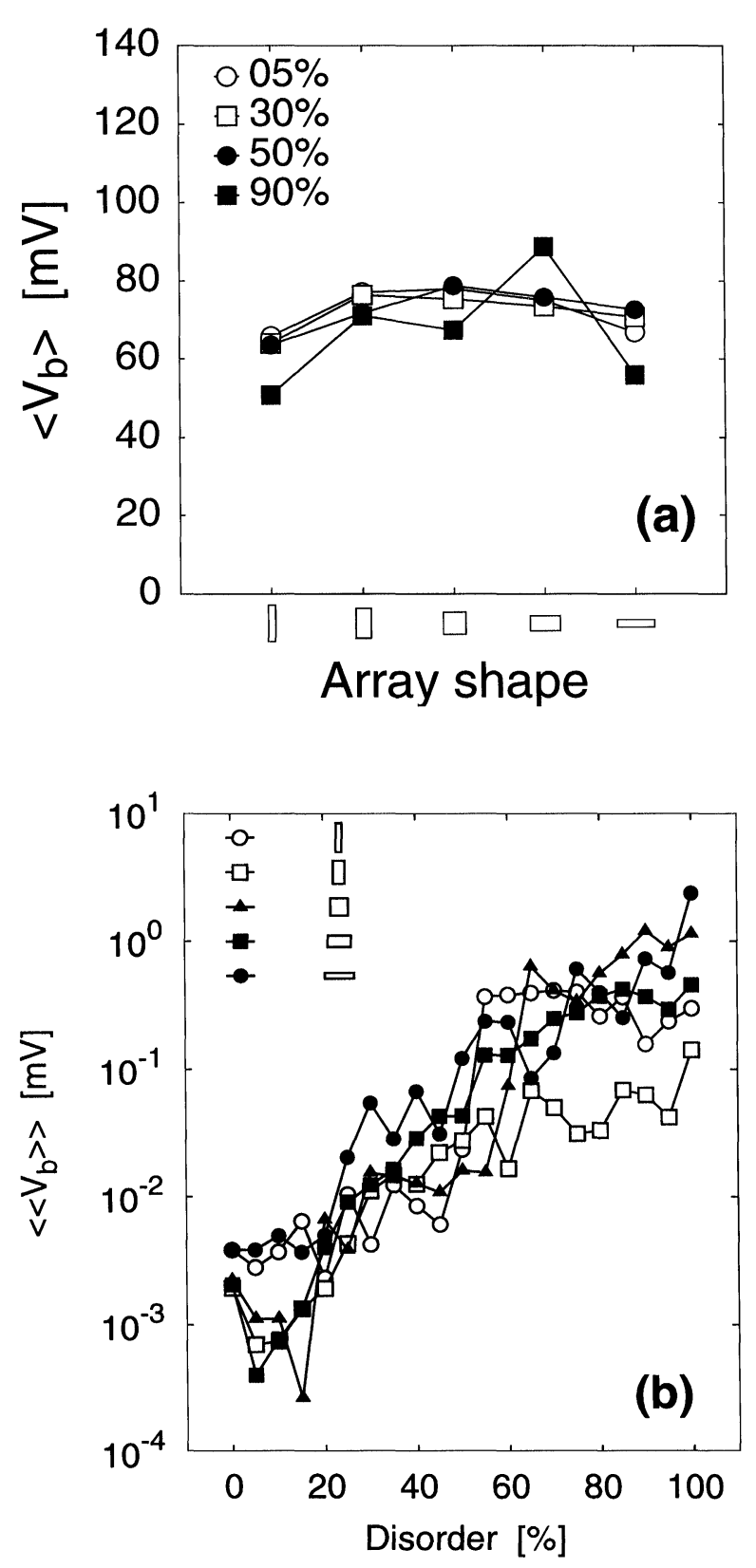

FIGURE 3 (a) $\left\langle V_{b}\right\rangle$ is plotted as a function of array shape for different levels of disorder and (b) $\left\langle\left\langle V_{b}\right\rangle\right\rangle$ is plotted as a function of disorder for different array shapes.

quantum dots nearby. On the other hand, wider arrays will have more competing tunneling paths, thus effectively reducing $\left\langle V_{b}\right\rangle$. For ordered arrays, on the other hand, both mechanisms show little effect and the $\left\langle V_{b}\right\rangle$ dependence is flat. Stepping through different disorder levels allows us to observe the onset of charge pinning and the concurrent transition from "mainly ordered" to "significantly disordered" arrays. This transition happens at high disorder $(>50 \%)$ only, which corresponds to a value of $\left\langle\left\langle V_{b}\right\rangle\right\rangle$ in the $\mathrm{mV}$-range. For the $90 \%$-curve pinning is effective for all but the $5 \times 1$ array. This emphasizes that pinning is much stronger in two-dimensional arrays.

For the $5 \times 2$ array and wider arrays at $90 \%$ disorder a consistent increase of $\left\langle V_{b}\right\rangle$ is found, however there is a significant difference between the values of the $5 \times 1$ array and the $5 \times 2$ array. This indicates that the observed pinning is a twodimensional process while in the case of the $5 \times 1$ array pinned charges act similarly to offset charges, namely reduce the blockade voltage. The proposed pinning process involves an electron and a hole in two parallel paths, which clog these paths for subsequent charges [12].

In Figure 2(a), the data corresponding to subpinning disorder show a slight increase of $\left\langle V_{b}\right\rangle$. This increase is solely attributed to capacitance effects independent of the level of disorder. The total capacitance of a dot, $\mathrm{C}_{\Sigma}$, depends on the arrangement of the other electrodes in its vicinity. Symmetric setups have lower total capacitances, which translates into higher blockade voltage. Since the wider the array the more symmetric the system is, a slight increase of $\left\langle V_{b}\right\rangle$ is anticipated.

Another example is the transition from the wide to the narrow array as shown in Figure 3. Here we plot $\left\langle\left\langle V_{b}\right\rangle\right\rangle$ as function of disorder with 20 different levels. Again, the value of $\left\langle\left\langle V_{b}\right\rangle\right\rangle$ is a fingerprint of the disorder level in the device, almost independent of the array geometry. Disorder induced charge pinning is observed for the $90 \%$-curve. The variation of the other curves with the array shape results from changing capacitances: for non-identical dots, the $1 \times 5$ array always displays a lower Coulomb blockade than longer arrays.

$\left\langle V_{b}\right\rangle$ as function of the array shape for disorder less than $90 \%$ shows a slight hump for the $3 \times 3$ array. This can be explained again with the argument of symmetry, see the discussion of 
Figure 2(a). Both the $1 \times 5$ array and the $5 \times 1$ array are rather asymmetric compared to the $3 \times 3$ array. Therefore we expect first a slight increase $(1 \times 5 \rightarrow 3 \times 3)$ and later a decrease $(3 \times 3 \rightarrow 5 \times 1)$ back to the original value of $\left\langle V_{b}\right\rangle$.

The coupling of the dots in the y-direction, perpendicular to the current flow, can be estimated in comparison to the one dimensional case, for which an analytic solution of $\left\langle V_{b}\right\rangle$ exists [13]. In the case of vanishing coupling, the Coulomb blockade voltage is the same for one- and twodimensional arrays of given length. Coupling can increase or decrease $\left\langle V_{b}\right\rangle$ as a result of charge pinning, or by providing more favorable current paths in two dimensions. In Figure 4 we study the perpendicular coupling. In this example we use square-like arrays from size $1 \times 1$ to $5 \times 5$ for different levels of size disorder. The results are compared to the solution of the one-dimensional case (dashed line). Then the blockade voltage depends on a critical size $N_{c}$ of the array, which in turn is given in terms of the inter-dot capacitance $C$ and the stray capacitances $C_{\mathrm{g}}$ of the array's dots. For the regular array we find $C=0.49 \mathrm{aF}$ and $\mathrm{C}_{\mathrm{g}}=1.26 \mathrm{aF}$ for our $5 \times 5 \mathrm{~nm}^{2}$ dots. This yields $N_{c}=2.5 \sqrt{C / C_{g}} \approx 1.6$.

Our data follow roughly the curve of the onedimensional case, i.e., they show a strong increase in $\left\langle V_{b}\right\rangle$ with size for small arrays and a saturation

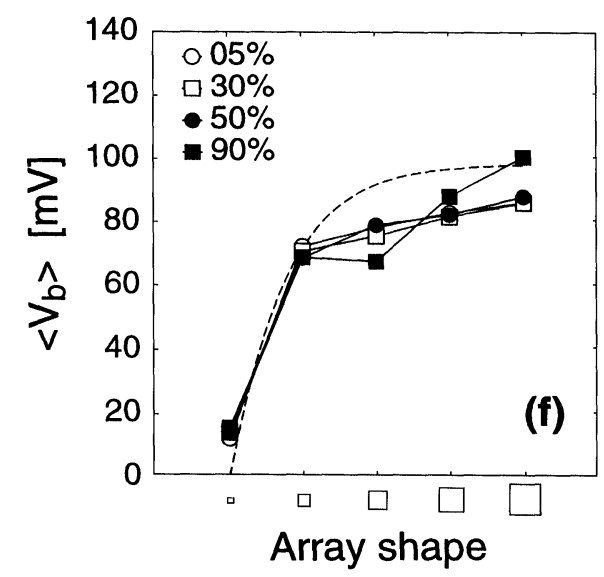

FIGURE 4 Array width dependence of $\left\langle V_{b}\right\rangle$ for different values of size disorder and different array geometry, respectively. for large ones. Saturation sets in already for the $2 \times 2$ array, in concurrence with the analytical result and almost independent of the level of disorder. Besides the $90 \%$ curve, which is influenced by charge pinning, the data show a saturation value of $\left\langle V_{b}\right\rangle$ which is smaller than the one-dimensional formula predicts. This is an indication of the coupling, which provides parallel tunneling paths. This does not work for the single dot, obviously, and the $2 \times 2$ array, where all dots are accessible from the external leads. Note, that the saturation value here is higher than $\left\langle V_{b}\right\rangle$ for the $5 \times 1$ array of Figure 2(a). This difference is once more attributed to the symmetry of the considered system. In detail, the square-like array will result in smaller capacitances and higher blockade voltage.

\subsection{Offset Charges and Array Width}

Figure 5 displays $\left\langle V_{b}\right\rangle$ and $\left\langle\left\langle V_{b}\right\rangle\right\rangle$ as function of the array width for different levels of the offset charge variation. $\left\langle\left\langle V_{b}\right\rangle\right\rangle$ Figure $5(\mathrm{~b})$, is almost constant. It does not show a clear dependence on the array width or the disorder level, except for the lowest level of disorder. This constant value is about $1 \mathrm{mV}$, allowing charge pinning in accordance with our study of size disorder, see Figure 2. Therefore we discuss the behavior of $\left\langle V_{b}\right\rangle$, Figure 5(a), in terms of charge pinning.

We observe a convergence of the $\left\langle V_{b}\right\rangle$ values for wider arrays, which is an averaging effect between different competing current channels. In contrast, for narrow arrays and high degree of disorder we
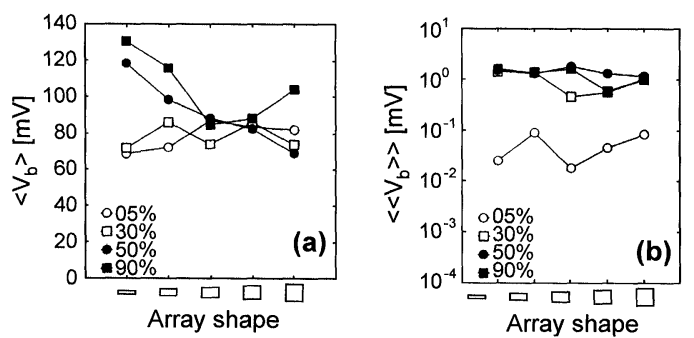

FIGURE 5 Array width dependence of $\left\langle V_{b}\right\rangle$ and $\left\langle\left\langle V_{b}\right\rangle\right\rangle$ for different levels of offset charge variation. 

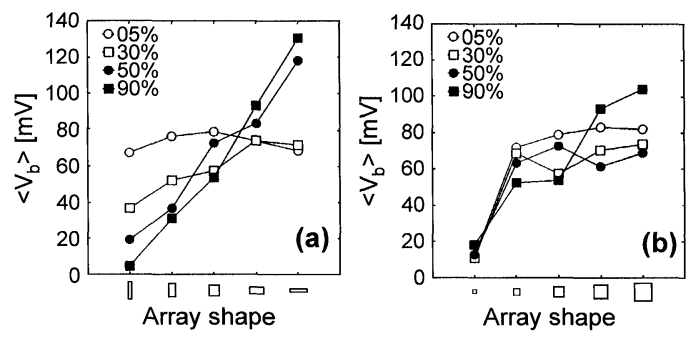

FIGURE $6\left\langle V_{b}\right\rangle$ for different array shapes and different levels of offset-charge variation.

see a drastic increase of $\left\langle V_{b}\right\rangle$ which is due to charge pinning. As the array becomes wider, charges can avoid these unfavorable paths.

In Figure 6 we concentrate on $\left\langle V_{b}\right\rangle$. For the transition from the short and wide to the long and narrow array we observe a strong increase in $\left\langle V_{B}\right\rangle$ for large disorder, but almost no variation for the regular case. Offset charges are well-known for their ability to suppress Coulomb blockade and this is observed for the wide array offering several transport channels. If only one of these channels is continuously open, CB cannot be observed. Increasing offset charge variation increases the chance of this happening. For the long and narrow array the situation is very different since there is no competition between transport channels. In this case charge pinning is rather likely to be observed in the remaining channels and $\left\langle V_{b}\right\rangle$ is increased.

In the other panel we apply again our analysis of the coupling perpendicular to the current flow. In general we find again a strong increase for small arrays and a saturation later on. The saturation value, however, is generally lower for higher disorder levels. Hence, coupling means more favorable tunneling paths again and this coupling increases with increasing disorder. The $90 \%$-curve shows again large $\left\langle V_{b}\right\rangle$ for large arrays, indicating a lack of beneficial tunneling paths.

\section{CONCLUSIONS}

In this paper we studied disorder in CB devices by means of simulation. The critical size $N_{c}$ of a quantum dot array or tunnel junction network proves useful for the description of the device behavior. Arrays smaller than $N_{c}$ show a reduced value of the average blockade voltage $\left\langle V_{b}\right\rangle$ and cannot be recommended for device application. Similarly short and wide arrays prove vulnerable to CB suppression by offset charges and shall be avoided. In long and narrow arrays, offset charges will result in charge pinning, which might change $V_{b}$ unpredictably. Therefore the conclusion is to use square-like $n \times n$ arrays of size $n \geq N_{c}$. Reliable device operation is also supported by the competition between different transport channels in those devices. In general, we found an exponential increase of the variation of the $\mathrm{CB}$ voltage, $\left\langle\left\langle V_{b}\right\rangle\right\rangle$ which is only weakly dependent on the array shape.

\section{Acknowledgment}

This work was performed within the ESPRIT MEL-ARI project FASEM (Fabrication and Architecture of Single-Electron Memories). We would like to thank S. Amakawa for helpful discussion.

\section{References}

[1] Grabert, H. and Devoret, M. H., Single Charge Tunneling: Coulomb Blockade Phenomena in Nanostructures, NATO ASI Series B: Physics. Vol 294 (Plenum, New York and London, 1992).

[2] Kauppinen, J. P. et al. (1998). "Coulomb Blockade Thermometer: Tests and Instrumentation”, Rev. Sci. Instrum., 69, 4166.

[3] Keller, M. W., Martinis, J. M., Zimmermannn, N. M. and Steinbach, A. H. (1996). "Accuracy of Electron Counting Using a 7-Junction Electron Pump", Appl. Phys. Lett., 69, 1804.

[4] Durrani, Z. A. K., Irvine, A. C., Ahmed, H. and Nakazato, K. (1999). "A Memory Cell with SingleElectron and Metal-Oxide-Semiconductor Transistor Integration", Appl. Phys. Lett., 74, 1293.

[5] Bakhvalov, N. S., Kazacha, G. S., Likharev, K. K. and Serdyukova, S. I. (1991). "Statistics and Dynamics of Single-Electron Solitons in 2-Dimensional Arrays of Ultrasmall Tunnel-Junctions", Physica B, 173, 319, preprint JINR-E11-90-177.

[6] Middleton, A. A. and Wingreen, N. S. (1993). "Collective Transport in Arrays of Small Metallic Dots", Phys. Rev. Lett., 71, 3198. 
[7] Rimberg, A. J., Ho, T. R. and Clarke, J. (1995). "Scaling Behavior in the Current-Voltage Characteristics of Oneand Two-Dimensional Arrays of Small Metallic Islands", Phys. Rev. Lett., 74, 4714.

[8] Delsing, P. et al. (1994). "Charge Solitons and Quantum Fluctuations in Two-Dimensional Arrays of Small Josephson Junctions", Phys. Rev. B, 50, 3959.

[9] Delsing, P. et al., "Two-Dimensional Arrays of Low Capacitance Tunnel Junctions: General Properties, Phase Transitions and Hall Effect", contribution to Euroschool on "Superconductivity in Networks and Mesoscopic Systems", held in Sienna, Italy (8-20 September, 1997), cond-mat/9805121 (unpublished).

[10] Müller, H. O., Katayama, K. and Mizuta, H. (1998). "Effects of Disorder on the Blockade Voltage of
Two-Dimensional Quantum Dot Arrays", J. Appl. Phys., 84, 5603.

[11] Ingold, G. L. and Nazarov, Y. V., "Charge Tunneling Rates in Ultrasmall Junctions", In: Single Charge Tunneling: Coulomb Blockade Phenomena in Nanostructures, NATO ASI Series B: Physics, Vol. 294, edited by Grabert, H. and Devoret, M. H. (Plenum, New York and London, 1992), pp. 21-107.

[12] Averin, D. V., Korotkov, A. N. and Nazarov, Yu. V. (1991). "Transport of Electron-Hole Pairs in Arrays of Small Tunnel Junctions", Phys. Rev. Lett., 66, 2818.

[13] Melsen, J. A., Hanke, U., Müller, H. O. and Chao, K. A. (1997). "Coulomb Blockade Threshold in Inhomogeneous One-Dimensional Arrays of Tunnel Junctions", Phys. Rev. $B, \mathbf{5 5}, 10638$, cond-mat/9611052. 

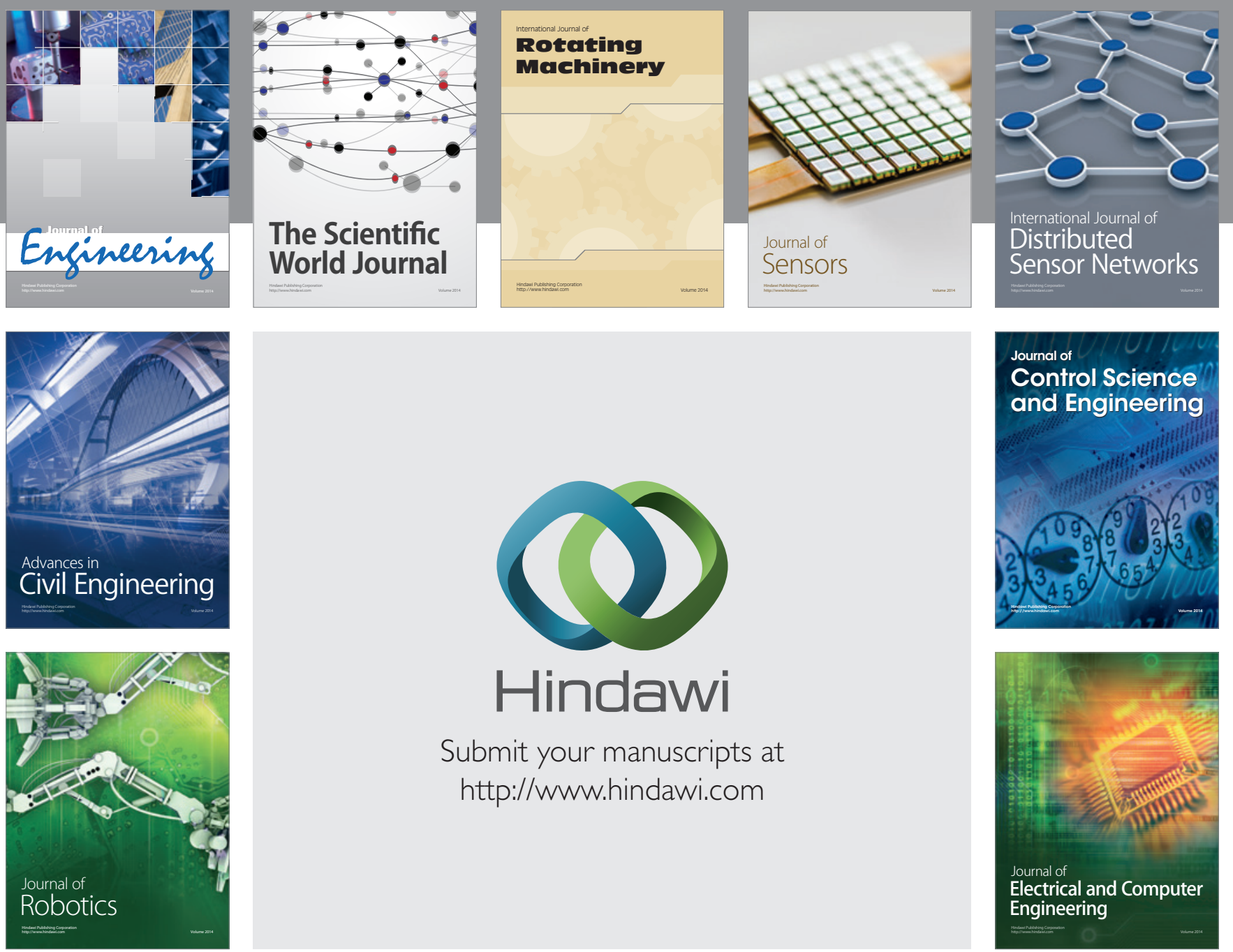

Submit your manuscripts at

http://www.hindawi.com
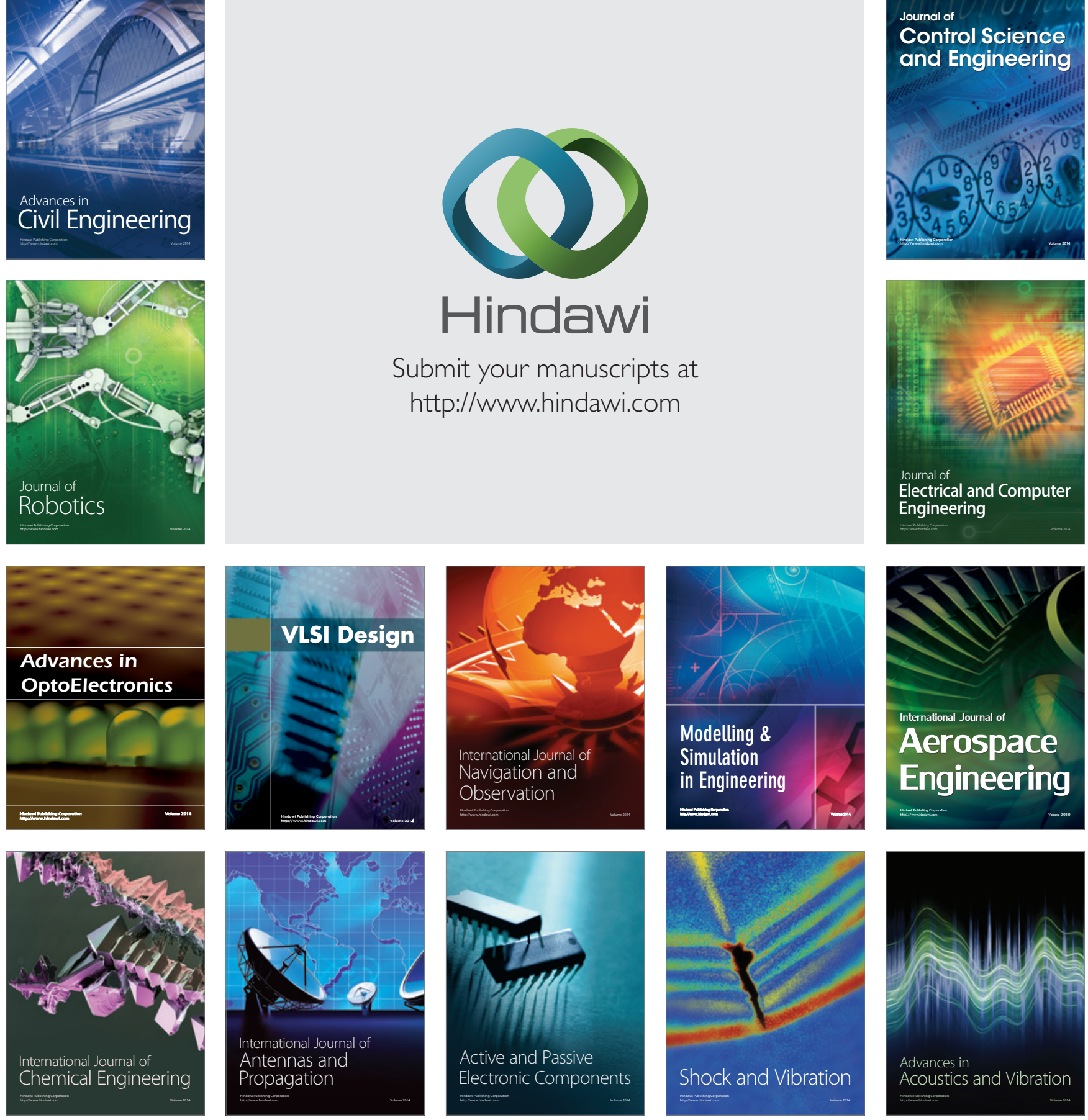